\title{
Modelling and Optimal Control of Ebola Virus Disease in the Presence of Treatment and Quarantine of Infectives
}

\author{
Herick Laiton Kayange ${ }^{1}$, Estomih Shedrack Massawe ${ }^{2,}$, , Daniel Oluwole Makinde ${ }^{3}$, \\ Lathika Sunil Immanuel $^{2}$ \\ ${ }^{1}$ Department of Mathematics, University of Dar es Salaam, Dar es Salaam, Tanzania \\ ${ }^{2}$ College of Engineering and Technology, St. Joseph University in Tanzania, Dar es Salaam, Tanzania \\ ${ }^{3}$ Faculty of Military Science, Stellenbosch University, Stellenbosch, South Africa
}

\section{Email address:}

herickkayange@gmail.com (H. L. Kayange), emassawe2@gmail.com (E. S. Massawe), makinded@gmail.com (D. O. Makinde), plathilintu.sunil@gmail.com (L. S. Lathika)

${ }^{*}$ Corresponding author

\section{To cite this article:}

Herick Laiton Kayange, Estomih Shedrack Massawe, Daniel Oluwole Makinde, Lathika Sunil Immanuel. Modelling and Optimal Control of Ebola Virus Disease in the Presence of Treatment and Quarantine of Infectives. International Journal of Systems Science and Applied Mathematics. Vol. 5, No. 4, 2020, pp. 43-53. doi: 10.11648/j.ijssam.20200504.12

Received: October 30, 2020; Accepted: November 11, 2020; Published: November 23, 2020

\begin{abstract}
In this paper, a non-linear mathematical model for the dynamics of Ebola virus diseases is formulated and analysed. The model has five classes namely susceptible human, exposed human, infected human, treated human and recovered human. Invariant region and positivity solution of the model are determined. Local stability analyses of disease free Equilibrium and endemic equilibrium are examined. The disease free equilibrium analysis is determined using Routh-Hurwitz criteria, whereby it is found to be locally stable if the reproduction number is less than one. Two control measures: control measure due to quarantine of exposed and susceptible individuals and control measure due to efficacy of treatment drug, used for treating Ebola virus disease Ebola victim are incorporated to the Ebola virus disease model. The control problem is then analysed in order to determine the optimal control. Numerical simulations for the model in the presence of control measures are finally performed. The results show that in the presence of optimal control, the Ebola virus disease can be eliminated in the Society. Furthermore, to minimize infections of Ebola virus disease, quarantine centres with skilled manpower must be prepared in advance so as to accommodate the significant number of exposed and susceptible individuals, in order to avoid further transmission in other areas out of quarantine centres. Also tracing of exposed and infected individuals must be efficiently done in order to quarantine the affected population and educate people on the transmission of the disease, symptoms and prevention measures in order to minimize human to human transmissions. Investing more on researches on new drugs which are effective in treating the Ebola virus disease victim is inevitable.
\end{abstract}

Keywords: Ebola, Control, Quarantine, Treatment, Infectives

\section{Introduction}

Ebola Virus Disease (formerly known as Ebola haemorrhagic fever) is a severe often fatal illness in humans. Ebola is a disease with haemorrhage bleedings. It usually affects human and non-human primates. Non-human primates include Monkeys, Gorillas and Chimpanzees [1]. Ebola virus comprises of five distinct species: Bundibugyo Ebolavirus (BDBV), Zaire Ebolavirus (EBOV), Reston Ebolavirus (RESTV), Sudan Ebolavirus (SUDV) and Tai Ebolavirus

\section{(TAFV) [2].}

Ebola is characterized by flu like symptoms which rapidly progress to vomiting, diarrhoea, rash, internal and external bleeding [3]. Ebola is introduced into human population through close contact with the blood, secretions, organs or other bodily fluids of infected animals. In Africa, infection has been spreading through the handling of infected chimpanzees, Gorillas, Fruitbats, Monkeys, Forest antelopes and porcupines found ill or dead in the Rain Forest. Ebola then spreads in the community through human to human transmission with infection resulting from direct contact (through scratched skin 
or muscular membrane). Burial ceremonies in which mourners have direct contact with the body of the deceased can also play a role in Transmission of Ebola [4]. An incubation period, a time interval from infection with virus to onset of symptoms is 2 to 21 days [1].

Supportive car-rehydration with oral intravenous fluids and treatment of specific symptoms improve survival from Ebola virus disease [1] The treatment of Ebola hemorrhagic fever infected patient consists mainly of palliative treatment and treatment to avoid cardiovascular collapse and renal insufficiency [5]. Immediate quarantine, treatment and management by the hospital can halt the spread of the outbreak if one is quick to act on a person who has been in contact with Ebola patient, or patient suspected of Ebola fever [6].

In dynamical systems, optimal control is the process of determining control and state trajectories of dynamical system over a period of time in order to maximize a given performance index [7]. Optimal Control Theory is applied to suggest the most effective mitigation theory to minimize number of individuals who become infected in the course of an infection while balancing vaccination and treatment applied to the models with various cost scenarios [8]. The control problem in dynamical systems is in the form of $\dot{x}(t)=g(t, x(t), u(t))$ where by, $t=$ time, $u(t)=$ the control variable and $x(t)=$ state variable. The control is measurable with $0 \leq u(t) \leq 1$. Both control and the state usually affect the goal which is called objective function [9].

The basic optimal control problems involves a cost functional or performance criterion say $J(u)$. which is of the form,

$$
J(u)=\int_{0}^{T} f(t, x, u) d t+\Phi(T)
$$

subject to the above differential equations and initial conditions. Such a minimizing control is called an optimal control problem. $f$ is a given real valued function and $\Phi$ is a continuous real-valued function. In the minimization case, the goal is to find an optimal control $u^{*}$ such that,

$$
J\left(u^{*}\right)=\underset{u}{\operatorname{Min}} J(u) .
$$

The principle technique for such an optimal control problem is to solve a set of "necessary conditions" that an optimal control and corresponding state variables must satisfy [10]. The necessary conditions is generated from the Hamiltonian $H$, which is defined as

$$
H=f(t, u(t), x(t))+\lambda g(t, u(t), x(t)) .
$$

$$
\text { Subject to } \frac{d g_{i}}{d t}=q\left(g_{i}(t), u(t), t\right)
$$

where $\lambda$ is an adjoint function [9].

The necessary conditions for maximizing/minimizing $H$ with respect to $u$ at $u^{*}$ are,

$$
\begin{gathered}
\left.\frac{\partial H}{\partial u}\right|_{u=u^{*}}=0, \rightarrow f_{u}+\lambda g_{u}=0 \\
\lambda^{\prime}=-\frac{\partial H}{\partial x}=0 \rightarrow \lambda^{\prime}=-\left(f_{x}+\lambda g_{x}\right)
\end{gathered}
$$

and

$$
\lambda(T)=0 \quad[9]
$$

It is then intended to optimise the quarantine of exposed and infected individuals and to optimise the efficacy of drug used for treating Ebola virus disease victim in order to minimize the infections of Ebola virus disease.

\section{Model Formulation}

Chowell et al. developed a Mathematical model to investigate the epidemics of Ebola, the basic reproduction number of Ebola and the effects of public measures, using the cases of Congo and Uganda [11].

DiekMann et al. conducted a study on mathematical modelling, simulation and optimal control of the 2014 Ebola outbreak in West Africa, whereby they introduced the control on vaccination rate of Ebola [12].

In this paper, a Mathematical model for Ebola Virus Disease in the presence of treatment and quarantine of infectives will be formulated and analysed. It is intended to extend the mathematical model by Chowell et al. who established a simple SEIR-model to elaborate the dynamics of Ebola virus disease [11]. The process will include recruitment rate of susceptible population, disease transmission rate by exposed population to susceptible population, natural death rate in each class, death due to the disease and possibility of the recovered population to be susceptible again. All these inputs are significant in transmission of Ebola virus disease. In modelling the dynamics of the disease, the population is divided into five subclasses namely, $S(t)$ : The Susceptible human, the number of people who are not yet affected by Ebola Virus Disease, $E(t)$ : The Exposed Human beings, the number of individuals who are affected by Ebola Virus Disease but not yet infectious, $I(t)$ : The Infected, number of individuals who are affected with Ebola Virus Disease who can spread that disease to the susceptible population, $T(t)$ : The number of people who are subjected to treatment procedure at time $t$ and $R(t)$ : The number of people who have recovered from Ebola Virus Disease.

Ebola virus disease is transmitted from the exposed population and infected population at the rates of $\beta_{1}$ and $\beta_{2}$ respectively. Therefore, the susceptible population is recruited at the exposed class at the rate of $\beta_{1} E+B_{2} I$. The exposed group gets infected and joins the infectious group at the rate of $\delta$, infectious joins the treated group at the rate of $\gamma$ whereby $m$ proportion of infectious group die due to Ebola virus disease, treated individuals get recovered at the rate of $b$, recovered population joins the susceptible class at rate of 
$a$. Natural death rate is constant $(\mu)$ in each of the five classes.

In formulating the model, the following assumptions are taken into consideration:-

Recruitment rate of susceptible humans is constant,

The Ebola virus disease patient cannot get immunity after recovering from the disease,

Ebola virus disease infection is proportional to the number of infectives,

All people affected by virus disease pass through the same disease process,

Ebola virus disease infection is possible through Human to Human only.

With $S$ the susceptible humans, $E$ the exposed human to Ebola virus, $I$ the infected human, $T$ the treatment group, $R$ the recovered group, $Q$ the recruitment rate of susceptible human, $\beta_{1}$ the disease transmission rate to susceptible human by exposed human, $\beta_{2}$ the disease transmission rate to the susceptible human by the infected human, $a$ the rate of progression to susceptible group from recovered group, $\mu$ the human natural death rate, $b$ the rate of progression of treated group to recovered group, $\gamma$ the rate of progression of infected to treated group, $\delta$, the rate of progression of exposed group to infected group, $m$ the death rate due to Ebola virus disease and taking into account the above considerations and assumptions, we have the following schematic flow diagram:

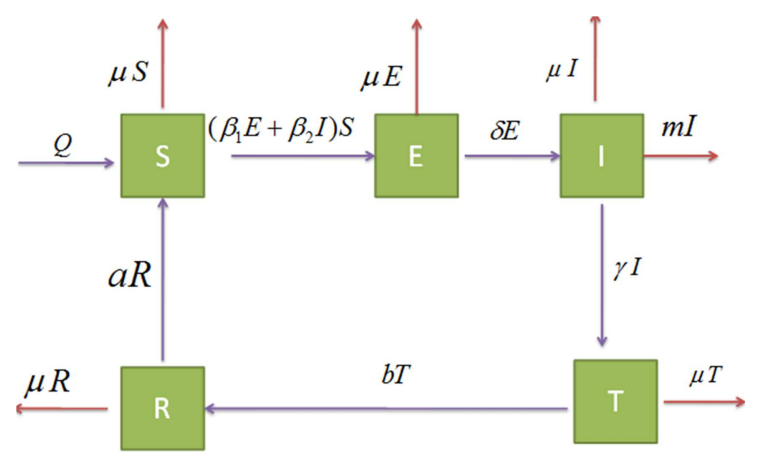

Figure 1. Compartmental representation of the model.

From the above flow diagram, the dynamics of the disease is governed by the following system of nonlinear ordinary differential equations:

$$
\begin{aligned}
& \frac{d S}{d t}=Q-\left(\beta_{1} E+\beta_{2} I\right) S+a R-\mu S \\
& \frac{d E}{d t}=\left(\beta_{1} E+\beta_{2} I\right) S-(\delta+\mu) E \\
& \frac{d I}{d t}=\delta E-(\gamma+m+\mu) I \\
& \frac{d T}{d t}=\gamma I-b T-\mu T
\end{aligned}
$$

$$
\frac{d R}{d t}=b T-(a+\mu) R
$$

where $S(0)=S_{0} \geq 0, \quad E(0)=E_{0} \geq 0, \quad I(0)=I_{0} \geq 0$, $T(0)=T_{0} \geq 0$ and $R(0)=R_{0} \geq 0$ are the initial conditions of the above model.

\section{Model Analysis}

The model system (1) will be analysed qualitatively to get insight to its dynamical features which give a better understanding of Ebola virus disease in the presence of treatment and quarantine of infectives. Threshold which governs elimination or persistence of Ebola virus disease in the presence of treatment and quarantine of infectives will be determined and analysed.

\subsection{Invariant Region}

Since the model (1) is Ebola model dealing with human population, we assume that all state variables and parameters of the model are positive. The model (1) will then be analysed in suitable feasible region where all state variables are positive. This region will be obtained by considering the following lemma:-

Lemma 1: The feasible region $\Omega$, contains the solution set $(S, E, I, T, R) \in \mathbb{R}^{5}$.

Proof:

If $N$ is the total population size, then

$$
N=S+E+I+T+R
$$

It follows that,

$$
\begin{aligned}
& \frac{d N}{d t}=\frac{d S}{d t}+\frac{d E}{d t}+\frac{d I}{d t}+\frac{d T}{d t}+\frac{d R}{d t} \\
& \frac{d N}{d t}=Q-\mu(N)-m I \\
& \frac{d N}{d t} \leq Q-\mu N \\
& N(t) \leq \frac{Q-(Q-\mu N(0)) e^{-\mu t}}{\mu}
\end{aligned}
$$

As $t \rightarrow \infty, N(t) \leq \frac{Q}{\mu}$

Since $N(t) \leq \frac{Q}{\mu}$, then the basic Mathematical model is well posed and it is mathematically relevant, therefore it is sufficient to study the dynamics of the epidemiological system (1) in the region $\Omega$.

\subsection{Positivity of the Solution}

Lemma 2: If the initial Solution of a dynamical model (1) is 
$\{S(t) \geq 0, E(t) \geq 0, I(t) \geq 0, T(t) \geq 0, R(t) \geq 0\} \in \Omega \frac{1}{2}$, then the solution set of dynamical model (1) is positive for all $t \geq 0$.

Proof:

Consider $\frac{d s}{d t}$ in the system (1):

$$
\frac{d S}{d t}=Q-\left(\beta_{1} E+\beta_{2} I\right) S+a R-\mu S
$$

Then

$$
\frac{d S}{d t} \geq-\mu S
$$

The inequality (3) has a solution

$$
S \geq e^{-\mu t}
$$

As

$$
t \rightarrow \infty, S \geq 0 .
$$

Similarly using the other equations of system (1), positivity of solutions can be established. Hence, all the solutions of the system (1) are positive for all $t>0$.

\subsection{Disease free Equilibrium Point}

The Equilibrium point of the system (1) can be established by setting $\frac{d S}{d t}=\frac{d E}{d t}=\frac{d I}{d t}=\frac{d T}{d t}=\frac{d R}{d t}=0$.

Then

$$
\begin{aligned}
Q-\left(\beta_{1} E^{*}+\beta_{2} I^{*}\right) S^{*}+a R^{*}-\mu S^{*} & =0 \\
\left(\beta_{1} E^{*}+\beta_{2} I^{*}\right) S^{*}-(\delta+\mu) E^{*} & =0 \\
\delta E^{*}-(\gamma+m+\mu) I^{*} & =0 \\
\gamma I^{*}-b T^{*}-\mu T^{*} & =0 \\
b T^{*}-(a+\mu) R^{*} & =0
\end{aligned}
$$

When there is no disease, the disease free equilibrium point can be established by setting $I=0$ in the system (4) followed by solving the resulting system of simultaneous equations to get $\mathrm{E}_{0}=(E, I, T, R)=\left(\frac{Q}{\mu}, 0,0,0,0\right)$. Thus the disease free equilibrium point is given by

$$
\mathrm{E}_{0}=\left(\frac{Q}{\mu}, 0,0,0,0\right) .
$$

\subsection{The Basic Reproduction Number}

The basic reproduction number $R_{0}$, defined as the number of Secondary cases which one case would produce in a completely susceptible population [12].

Next generation matrix method is the natural basis for the definition and calculation of $R_{0}$, Where finitely many different categories of individuals are recognized [12] $R_{0}$ is the spectral radius of the next generation Matrix [16]. Next generation matrix $(G)$ is given by:

$$
\mathrm{G}=\mathrm{FV}^{-1}
$$

where $\mathrm{F}=\frac{\partial F_{i}\left(E_{0}\right)}{\partial x_{j}}, \mathrm{~V}=\frac{\partial V_{i}\left(E_{0}\right)}{\partial x_{j}}$ and $E_{0}$ is the disease free equilibrium point.

$F_{i}$ is the rate at which new infections appears at compartment $i$ and $V_{i}$ is the removal of individuals among different classes. Also $V_{i}=V_{i}^{-}-V_{i}^{+}$whereby $V_{i}^{+}$is rate of movement of individuals into the compartment $i$ and $V_{i}^{-}$is rate of movement of individuals out of compartment $i$.

Considering the equation (6),

Since,

$$
\mathrm{F}_{i}=\left(\begin{array}{l}
\left(\beta_{1} E+\beta_{2} I\right) S \\
0
\end{array}\right)
$$

then,

$$
\mathrm{F}=\frac{\partial F_{i}\left(E_{0}\right)}{\partial x_{j}}=\left(\begin{array}{ll}
\frac{\partial F_{1}}{\partial E} & \frac{\partial F_{1}}{\partial I} \\
\frac{\partial F_{2}}{\partial E} & \frac{\partial F_{2}}{\partial I}
\end{array}\right) .
$$

This can be rewritten as

$$
\mathrm{F}=\left(\begin{array}{cc}
\beta_{1} S & \beta_{2} S \\
0 & 0
\end{array}\right)
$$

If $\mathrm{V}_{i}$ is the movement of individuals out of each compartment $i$, then

$$
\mathrm{V}_{i}=\left(\begin{array}{l}
(\delta+\mu) E \\
(\gamma+m+\mu) I-\delta E
\end{array}\right)
$$

Let $V$ be the Jacobian of $\mathrm{V}_{i}$. Then

$$
\mathrm{V}=\frac{\partial V_{i}\left(E_{0}\right)}{\partial X_{j}}=\left(\begin{array}{ll}
\frac{\partial V_{1}}{\partial E} & \frac{\partial V_{2}}{\partial I} \\
\frac{\partial V_{1}}{\partial E} & \frac{\partial V_{2}}{\partial I}
\end{array}\right)=\left(\begin{array}{cc}
(\delta+\mu) & 0 \\
-\delta & (\gamma+m+\mu
\end{array}\right)
$$

Consequently

$$
\mathrm{V}^{-1}=\left(\begin{array}{cc}
\frac{1}{\delta+\mu} & 0 \\
\frac{\delta}{(\gamma+m+\mu)(\delta+\mu)} & \frac{1}{(\gamma+m+\mu)}
\end{array}\right)
$$


Then

$$
\mathrm{G}=\mathrm{FV}^{-1}=\left(\begin{array}{cc}
\frac{Q \beta_{1}}{\mu(\delta+\mu)}+\frac{Q \delta \beta_{2}}{\mu(\gamma+m+\mu)(\delta+\mu)} & \frac{Q \beta_{2}}{\mu(\gamma+m+\mu)} \\
0 & 0
\end{array}\right)
$$

The eigenvalues of $\mathrm{G}$ are given by

$$
\left(0, \frac{Q \beta_{1}}{\mu(\delta+\mu)}+\frac{Q \delta \beta_{2}}{\mu(\gamma+m+\mu)(\delta+\mu)}\right) .
$$

Therefore

$$
R_{0}=\frac{Q \beta_{1}}{\mu(\delta+\mu)}+\frac{Q \delta \beta_{2}}{\mu(\gamma+m+\mu)(\delta+\mu)}
$$

or

$$
R_{0}=\frac{Q\left((\gamma+m+\mu) \beta_{1}+\delta \beta_{2}\right)}{\mu(\gamma+m+\mu)(\delta+\mu)}
$$

\subsection{Local Stability Analysis of the Disease Free Equilibrium}

Theorem 1: The disease free equilibrium point is locally asymptotic stable if the basic reproduction number is less than one and unstable if the basic reproduction number is greater than one $\left(R_{0}>1\right)$ [13].

In epidemiological point of view, it means that the spread of Ebola virus disease can be eradicated whenever the reproduction number is less than one $\left(R_{0}<1\right)$.

In order to determine the stability of the disease free equilibrium point, it is necessary to linearize the system of differential equation (1), so as to find an approximation of the system at the disease free equilibrium point.

We write the system as

$$
f(\lambda)=(\lambda+\mu)(a+\lambda+\mu)(-\lambda-\mu-b)\left(\mu(\mu+m+\gamma+\lambda)(\delta+\lambda+\mu)-Q\left((\mu+m+\gamma+\lambda)\left(\beta_{1}+\delta \beta_{2}\right)\right)\right)=0
$$

Theorem 2: The disease free equilibrium is locally asymptotic stable if the eigenvalues of the Jacobian matrix has negative real parts [14].

The first three eigenvalues are:

$$
\lambda_{1}=-\mu<0, \lambda_{2}=-a-\mu<0
$$

and

$$
\lambda_{3}=-\mu-b<0
$$

Since the first three eigenvalues have negative real parts, Routh-hurwitz criteria will be used in the fourth factor, as follows:

$$
\mu(\mu+m+\gamma+\lambda)(\delta+\lambda+\mu)-Q(\mu(\mu+m+\gamma+\lambda))\left(\beta_{1}+\delta \beta_{2}\right)=0
$$

Equation (16) can be written as

$$
\lambda^{2}+A \lambda+B=0
$$

$$
\begin{aligned}
& g_{1}=Q-\left(\beta_{1} E+\beta_{2} I\right) S+a R-\mu S \\
& g_{2}=\left(\beta_{1} E+\beta_{2} I\right) S-(\delta+\mu) E \\
& g_{3}=\delta E-(\gamma+m+\mu) I \\
& g_{4}=\gamma I-b T-\mu T \\
& g_{5}=b T-(a+\mu) R
\end{aligned}
$$

The Jacobian Matrix of the system (12) is

$$
\mathrm{J}=\left(\begin{array}{ccccc}
-\left(\beta_{1} E+\beta_{2} I\right)-\mu & -\beta_{1} S & -\beta_{2} S & 0 & a \\
\left(\beta_{1} E+\beta_{2} I\right) & \beta_{1} S-(\delta+\mu) & \beta_{2} S & 0 & 0 \\
0 & \delta & -(\gamma+m+\mu) & 0 & 0 \\
0 & 0 & \gamma & -b-\mu & 0 \\
0 & 0 & 0 & b & -(a+\mu)
\end{array}\right)
$$

At the disease free equilibrium point $\left(\frac{Q}{\mu}, 0,0,0,0\right)$, the system (13) becomes

$$
\mathrm{J}=\left(\begin{array}{ccccc}
-\mu & \frac{-\beta_{1} Q}{\mu} & \frac{-\beta_{2} Q}{\mu} & 0 & a \\
0 & \frac{\beta_{1} Q}{\mu}-(\delta+\mu) & \frac{\beta_{2} Q}{\mu} & 0 & 0 \\
0 & \delta & -(\gamma+m+\mu) & 0 & 0 \\
0 & 0 & \gamma & -b-\mu & 0 \\
0 & 0 & 0 & b & -(a+\mu)
\end{array}\right)
$$

If $\lambda$ is an eigenvalue of the Jacobian matrix $\mathrm{J}$, then the characteristic equation of Matrix $\mathrm{J}$ is where,

$$
A=\mu(\mu+\gamma+m)+\mu(\delta+\mu)-Q
$$

and

$$
B=\mu(\mu+\gamma+m)(\delta+\mu)-Q\left(\mu(\mu+\gamma+m) \beta_{1}+\delta \beta_{2}\right)
$$$$
A>0 \text { if } \mu(\mu+\gamma+m)+\mu \delta+\mu(\delta+\mu)>Q
$$

$$
B>0 \text { if }
$$

$$
\mu(\mu+\gamma+m)(\delta+\mu)>-Q\left(\mu(\mu+\gamma+m) \beta_{1}+\delta \beta_{2}\right)
$$

or

$$
\frac{\left.Q\left(\mu(\mu+\gamma+m) \beta_{1}+\delta \beta_{2}\right)\right)}{\mu(\mu+\gamma+m)(\delta+\mu)}<1
$$


But $R_{0}=\frac{\left.Q\left(\mu(\mu+\gamma+m) \beta_{1}+\delta \beta_{2}\right)\right)}{\mu(\mu+\gamma+m)(\delta+\mu)}$.

Hence

$$
R_{0}<1
$$

Therefore $R_{0}<1$ if $B>0$ and $A>0$. Then by Routh-Hurwitz criteria the disease free equilibrium of the system (1) is locally asymptotically stable.

\subsubsection{Existence of Endemic Equilibrium}

Lemma 3: There exists a positive endemic equilibrium point if and only if $R_{0}>1$.

Proof:

Endemic equilibrium point for the system (1) can be determined by setting, $\frac{d S}{d t}=\frac{d E}{d t}=\frac{d I}{d t}=\frac{d T}{d t}=\frac{d R}{d t}=0$, if $I \neq 0$.

This gives the endemic equilibrium point $\left(S^{*}, E^{*}, I^{*}, T^{*}, R^{*}\right)$ as

$$
\begin{gathered}
S^{*}=\frac{Q}{\mu R_{0}} \\
E^{*}=\frac{Q(\gamma+m+\mu)\left(R_{0}-1\right)(a+\mu)(b+\mu)}{R_{0}((\delta+\mu)(\gamma+m+\mu)(a+\mu)(b+\mu)-a b \delta \gamma)} \\
I^{*}=\frac{Q\left(R_{0}-1\right) \delta(a+\mu)(b+\mu)}{R_{0}((\delta+\mu)(\gamma+m+\mu)(a+\mu)(b+\mu)-a b \delta \gamma)} \\
T^{*}=\frac{Q \gamma\left(R_{0}-1\right) \delta(a+\mu)}{R_{0}((\delta+\mu)(\gamma+m+\mu)(a+\mu)(b+\mu)-a b \delta \gamma)} \\
R^{*}=\frac{\left(R_{0}-1\right) Q b \gamma \delta}{R_{0}((\delta+\mu)(\gamma+m+\mu)(a+\mu)(b+\mu)-a b \delta \gamma)} .
\end{gathered}
$$

Therefore there is only one positive endemic equilibrium point, which exists for $R_{0}>1$.

For $I^{*}$ :

$$
\frac{d I^{*}}{d R_{0}}=\frac{Q \delta(a+\mu)(b+\mu)}{R_{0}^{2}((\delta+\mu)(\gamma+m+\mu)(a+\mu)(b+\mu)-a b \delta \gamma)}
$$

Therefore $\frac{d I^{*}}{d R_{0}}>0$. This implies that $R_{0}>1$ increases the value of positive $I^{*}$ and at $R_{0}=1, I^{*}=0$.

\subsubsection{Global Stability Analysis of Endemic Equilibrium}

Lemma 4: The endemic equilibrium of the mathematical model (1) is globally asymptotically stable if $R_{0}>1$ and unstable if $R_{0} \leq 1$.

Proof:

Using the constructed Lyapunov function as suggested by [15], the global stability of Endemic equilibrium can be established by defining the Lyapunov function as

$$
\begin{gathered}
L\left(S^{*}, E^{*}, I^{*}, T^{*}, R^{*}\right)=\left(S-S^{*}-S^{*} \log \frac{S^{*}}{S}\right) \\
+\left(E-E^{*}-E^{*} \log \frac{E^{*}}{E}\right) \\
+\left(I-I^{*}-I^{*} \log \frac{I^{*}}{I}\right)+\left(T-T^{*}-T^{*} \log \frac{T^{*}}{T}\right) \\
+\left(R-R^{*}-R^{*} \log \frac{R^{*}}{R}\right) .
\end{gathered}
$$

It can be shown that

$$
\frac{d L}{d t}=X-Y
$$

where

$$
\begin{gathered}
X=Q+\beta_{1} E^{*} \frac{\left(S-S^{*}\right)^{2}}{S}+\beta_{2} I^{*} \frac{\left(S-S^{*}\right)^{2}}{S}+a R^{*}+a R^{*} \frac{S^{*}}{S} \\
+\beta_{1} S^{*} \frac{\left(E-E^{*}\right)^{2}}{E}+\beta_{2} I S+\beta_{2} I \frac{E^{*} S^{*}}{E} \\
+\beta_{2} E^{*} S+\delta E+\frac{\delta I^{*} E^{*}}{I} \\
+\gamma I+\gamma I^{*} \frac{T^{*}}{T}+b T+b T \frac{R^{*}}{R}, \\
Y=Q \frac{S^{*}}{S}+\beta_{1} E \frac{\left(S-S^{*}\right)^{2}}{S}+\beta_{2} I \frac{\left(S-S^{*}\right)^{2}}{S}+a R \frac{S^{*}}{S}+a R^{*} \\
+\mu \frac{\left(S-S^{*}\right)^{2}}{S}+\beta_{1} S^{*} \frac{\left(E-E^{*}\right)}{E}+\beta_{2} I S^{*}+\beta_{2} I \frac{E^{*} S}{E} \\
+\beta_{2} I S+\beta_{2} E^{*} S^{*}+(\delta+\mu) \frac{\left(E-E^{*}\right)^{2}}{E}+\delta E^{*}+\frac{\delta I^{*} E^{*}}{I} \\
-(\gamma+m+\mu) \frac{\left(I-I^{*}\right)^{2}}{I}+\gamma I^{*} \frac{T^{*}}{T}+\gamma I^{*}+b T \frac{R^{*}}{R}+(a+\mu) \frac{\left(R-R^{*}\right)^{2}}{R} .(23)
\end{gathered}
$$

If $X<Y$ we obtain $\frac{d L}{d t}<0 . \frac{d L}{d t}=0$ if and only if $S=S^{*}, E=E^{*}, I=I^{*}, T=T^{*}$ and $R=R^{*}$.

Then the largest compact invariant set $\left\{\left(S^{*}, E^{*}, I^{*}, T^{*}, R^{*}\right) \in \Omega: \frac{d L}{d t}=0\right\}$ is the singleton $\left\{E^{*}\right\}$,

where $E^{*}$ is the endemic equilibrium. Therefore by Lassale Invariance principle, $E^{*}$ is globally asymptotically stable if $X<Y$.

\section{Model with Control}

\subsection{Introduction}

In this section, the mathematical model (1) is extended by incorporating control variables $u_{1}(t)$ and $u_{2}(t)$, where

$u_{1}(t)=$ Control measure due to quarantine of exposed and 
infected humans,

$u_{2}(t)=$ Control measure due to efficacy of treatment of drug used for Ebola virus victims.

Then the model becomes

$$
\begin{aligned}
& \frac{d S}{d t}=Q-\left(1-u_{1}\right)\left(\beta_{1} E+\beta_{2} I\right) S+a R-\mu S \\
& \frac{d E}{d t}=\left(1-u_{1}\right)\left(\beta_{1} E+\beta_{2} I\right) S-(\delta+\mu) E \\
& \frac{d I}{d t}=\delta E-(\gamma+m+\mu) I \\
& \frac{d T}{d t}=\gamma I-\left(1-u_{2}\right) b T-\mu T \\
& \frac{d R}{d t}=\left(1-u_{2}\right) b T-(a+\mu) R
\end{aligned}
$$

where the control functions are bounded i.e. $0 \leq u_{1}(t) \leq 1$ and $0 \leq u_{2}(t) \leq 1$.

\subsection{Analysis of the Ebola Optimal Control Problem}

Here we aim at minimizing the infections of Ebola Virus disease, while optimizing $u_{1}(t)$ (treatment and Quarantine of individuals) and $u_{2}(t)$ (efficacy of treatment drug used for treating Ebola virus disease victims). In order to minimize infection, it is required to minimize the objective function

$$
J\left(u_{1}, u_{2}\right)=\int_{t_{0}}^{T}\left(A_{1} S(t)+A_{2} E(t)+A_{3} T(t)+A_{4} R(t)+\frac{1}{2} \sum_{i=1}^{2} B_{i} u_{i}^{2}\right) d t
$$

subject to control problem $x^{\prime}(t)=g\left(t, x(t), u_{1}(t), u_{2}(t)\right)$, with $x\left(t_{0}\right)=x_{0}$ and $x(T)$ free variable. $B_{i}$ 's are relative costs of intervention over $\left[t_{0}, T\right]$, The constants $A_{1}, A_{2}, A_{3}$ and $A_{4}$ are positive weights. It is then intended to find optimal controls $u_{1}^{*}$ and $u_{2}^{*}$, such that $J\left(u_{1}^{*}, u_{2}^{*}\right)=\min J\left(u_{1}, u_{2}\right)$, where $0 \leq u_{1} \leq 1$ and $0 \leq u_{2} \leq 1$

The Pontryagin maximum principle will be used to suggest the necessary optimal conditions, by forming Hamiltonian function,

$$
\begin{array}{r}
H=A_{1} S(t)+A_{2} E(t)+A_{3} T(t)+A_{4} R(t) \\
+\frac{1}{2} \sum_{i=1}^{2} B_{i} u_{i}^{2}+\sum_{i=1}^{5} \lambda_{i} g_{i}\left(x, u_{1}(t), u_{2}(t)\right)
\end{array}
$$

where $g_{i}\left(x, u_{1}(t), u_{2}(t), t\right)$ are the equations on the right hand side of the Ordinary differential equation of Control problem and $\lambda_{i}$ are co-state variables with $i=1,2,3,4$ and 5 .

Theorem 3: There exists a pair of optimal controls $\left(u_{1}^{*}, u_{2}^{*}\right)$ and optimal solution $\left(S^{*}, E^{*}, I^{*}, T^{*}, R^{*}\right)$ that minimizes $J\left(u_{1}, u_{2}\right)$ also there exist adjoint functions $\lambda_{i}(t)$ for $i=1,2,3,4$ and 5 for Hamiltonian $(H)$,

$$
\begin{array}{cc}
H=A_{1} S(t)+ & A_{2} E(t)+A_{3} T(t)+A_{4} R(t)+\frac{1}{2} B_{1} u_{1}^{*}+\frac{1}{2} B_{1} u_{2}^{*}+\lambda_{1}\left(Q-\left(1-u_{1}\right)\left(\beta_{1} E+\beta_{2} I\right) S+a R-\mu S\right)+\lambda_{2}\left(\left(1-u_{1}\right)\left(\beta_{1} E+\beta_{2} I\right)\right) S \\
-(\delta & +\mu) E+\lambda_{3}(\delta E-(\gamma+m+\mu) I) \\
& +\lambda_{4}\left(\gamma I-\left(1-u_{2}\right) b T-\mu T\right) \\
& +\lambda_{5}\left(\left(1-u_{2}\right) b T-(a+\mu) R\right)
\end{array}
$$

The Transversality conditions are

Such that,

$$
\lambda_{i}(T)=0, \text { for } i=1,2,3,4 \text { and } 5 .
$$

The pair of Optimal controls $\left(u_{1}^{*}(t), u_{2}^{*}(t)\right)$ is obtained by solving for $u_{1}(t)$ and $u_{2}(t)$ in optimality conditions

$$
\begin{gathered}
\left(\frac{\partial H}{\partial u_{1}}=0\right) \text { and }\left(\frac{\partial H}{\partial u_{2}}\right) \text { as suggested by [19]. } \\
\frac{\partial H}{\partial u_{1}}=B_{1} u_{1}+\lambda_{1}\left(\beta_{1} E+\beta_{2} I\right) S 1 \\
-\lambda_{2}\left(\beta_{1} E+\beta_{2} I\right) S=0
\end{gathered}
$$

$\frac{d \lambda_{3}}{d t}=-\frac{\partial H}{\partial I}=\lambda_{1}\left(1-u_{1}\right) \beta_{2} S-\lambda_{2}\left(1-u_{1}\right) \beta_{2} S+\lambda_{3}(\gamma+m+\mu)-\lambda_{4} \delta$ 


$$
\frac{\partial H}{\partial u_{2}}=B_{2} u_{2}+\lambda_{4} b T-\lambda_{5} b T=0
$$

Then

$$
\begin{gathered}
\bar{u}_{1}=\frac{\left(\beta_{1} E+\beta_{2} I\right) S\left(\lambda_{2}-\lambda_{1}\right)}{B_{1}} \\
\bar{u}_{2}=\frac{b T\left(\lambda_{5}-\lambda_{4}\right)}{B_{2}}
\end{gathered}
$$

Consequently,

$$
\begin{gathered}
u_{1}^{*}=\max \left\{0, \min \left(1, \frac{\left(\beta_{1} E+\beta_{2} I\right) S\left(\lambda_{2}-\lambda_{1}\right)}{B_{1}}\right)\right\}, \\
u_{2}^{*}=\max \left\{0, \min \left(1, \frac{b T\left(\lambda_{5}-\lambda_{4}\right)}{B_{2}}\right)\right\} .
\end{gathered}
$$

have

$$
u_{1}^{*}=\left\{\begin{array}{l}
0, \text { if } \bar{u}_{1} \leq 0 \\
\bar{u}_{1}, \text { if } 0<\bar{u}_{1}<1 \\
1, \text { if } \bar{u}_{1} \geq 1
\end{array} \quad u_{2}^{*}=\left\{\begin{array}{l}
0, \text { if } \bar{u}_{2} \leq 0 \\
\bar{u}_{2}, \text { if } 0<\bar{u}_{2}<1 \\
1, \text { if } \bar{u}_{2} \geq 1
\end{array}\right.\right.
$$

\section{Numerical Simulations of the Model with Control}

In this section, we illustrate the analytical results of the study by carrying out numerical simulations of the model with control i.e. model system (23) using the set of estimated parameter values given in the Table 1 below.

Table 1. Parameter values used for Model Simulation.

\begin{tabular}{lll}
\hline Parameter & Value(per month) & Source \\
\hline$\beta_{1}$ & 0.16 & {$[18]$} \\
$\beta_{2}$ & 0.12 & Estimated \\
$\mu$ & 0.1 & Estimated \\
$m$ & 0.5 & {$[18]$} \\
$\gamma$ & 0.3086 & {$[18]$} \\
$\delta$ & 0.83 & {$[18]$} \\
$b$ & 0.063 & {$[18]$} \\
$a$ & 0.82 & Estimated \\
$Q$ & 40 & Estimated \\
\hline
\end{tabular}

Initial values are estimated as

$S(0)=30, E(0)=20, I(0)=18, T(0)=17$ and $R(0)=12$.

The weights $A_{i}=B_{i}=100$, for $i=1,2,3,4$ and 5 are used, in order to show the effect of optimizing control measures in a control problem (23) when,

No control is applied to a system of equations,

Only a control measure due to efficacy drug used for treating an Ebola virus disease victim $\left(u_{2}\right)$ is applied,

Only a control measure due to quarantine of exposed and susceptible individuals $\left(u_{1}\right)$ is used,

Both controls, which are $u_{1}$ (control measure due to quarantine of exposed and susceptible individuals) and $u_{2}$ (control measure due to efficacy drug used for treating an Ebola virus disease victim) are used in control problem.

Figure 2 below shows the simulation of Ebola control problem, when there is no control applied, that is $u_{1}=u_{2}=0$.

Figure 2(a), shows that when there is no control, the number of susceptibles is reduced with time due to removal by infections. Figure 2(b) shows that the exposed individuals increase with time before being reduced by removal of exposed individuals to infected population. Figure 2(c) shows that infected population increases with time. Figure 2(d) shows that treated population increases with time and figure 2(e) shows that recovered individuals are reduced with time.
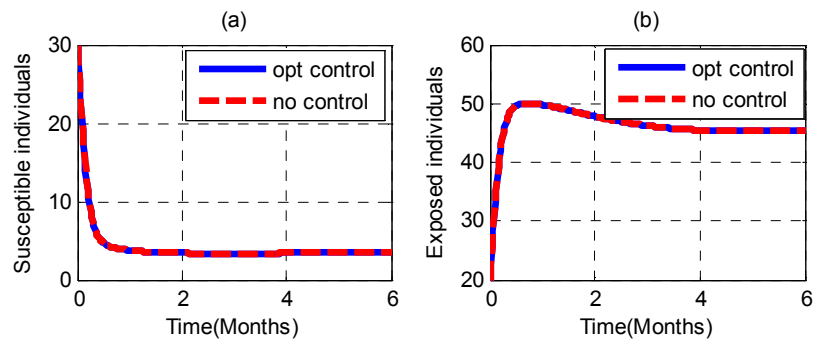

(c)
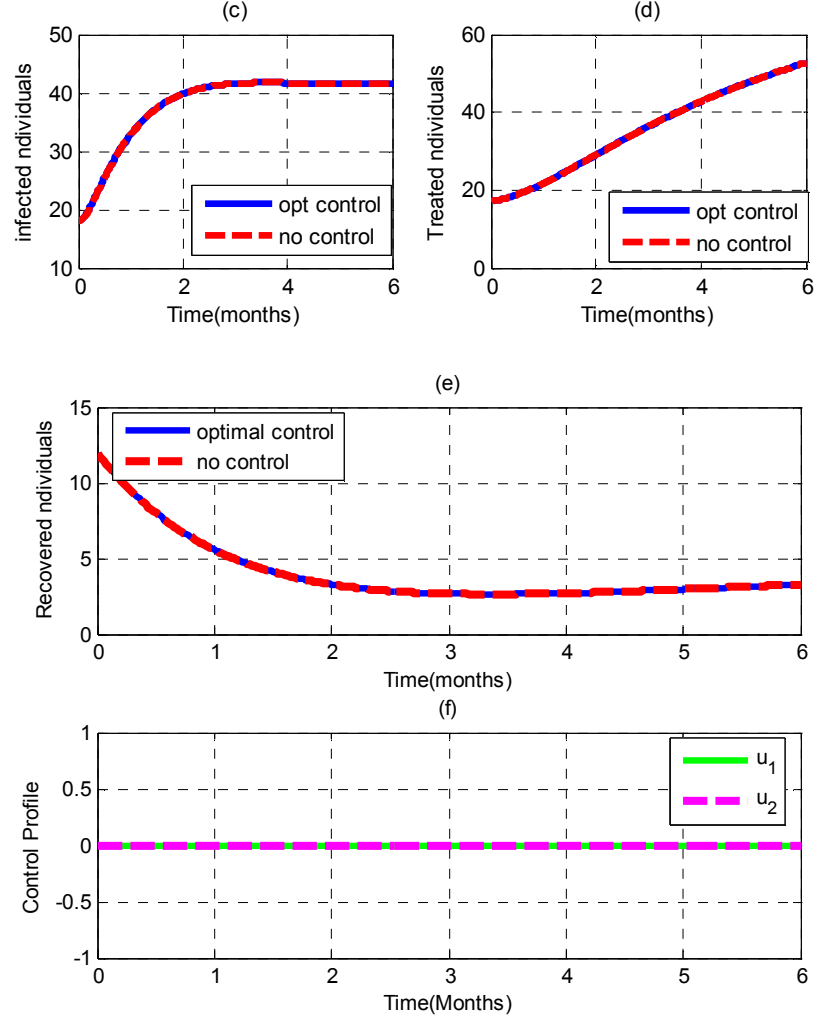

Figure 2. Simulation of Ebola control problem, when there is no control applied, that is $u_{1}=u_{2}=0$.

Therefore the disease will persist in the population for a long time, until some interventions strategies are introduced in the population.

Figures 3 below show the simulation of an objective function for Ebola virus disease when only a control 
measure $\left(u_{1}\right)$ due to quarantine of exposed and susceptible individuals is applied.

Figure 3(a) shows that susceptible population increases with time. Figure 3(b) shows that the exposed population decreases with time. Figure 3(c) shows that the infected population increases with time. Figure 3(d) shows that the treated population decreases with time due to reduction of infected population with time. Figure 3(e) shows that the recovered population will be decreases with time due to reduction of treated population with time. Figure 3(f) shows the control profile whereby $u_{1}$ (control measure due to quarantine of exposed and susceptible individuals) is set to the maximum and there is no control of efficacy drug used for treating Ebola virus disease victim $\left(u_{2}=0\right)$.

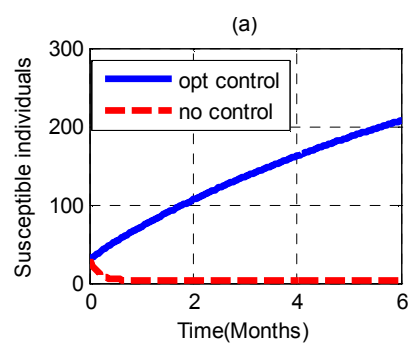

(c)
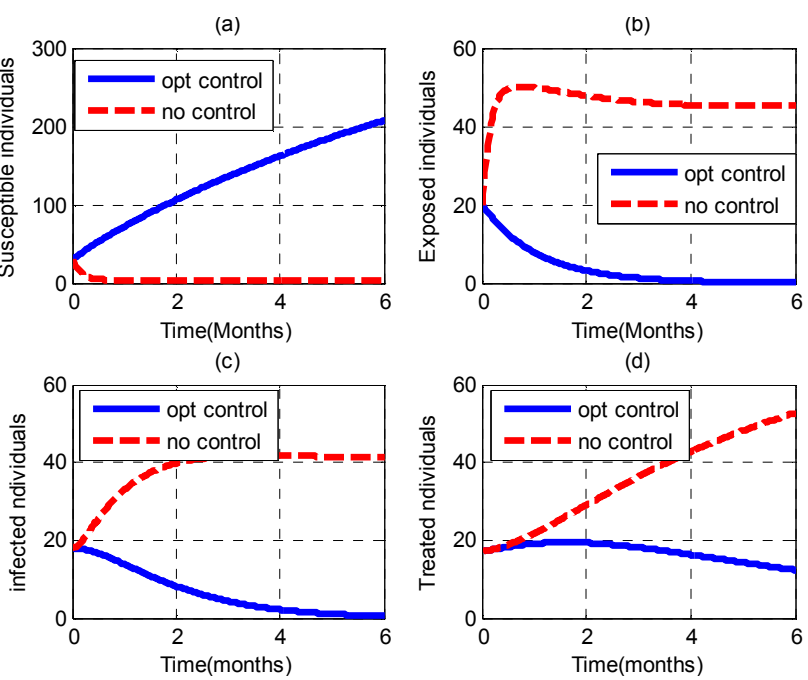

(d)

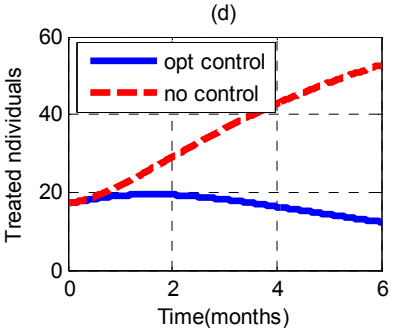

(e)

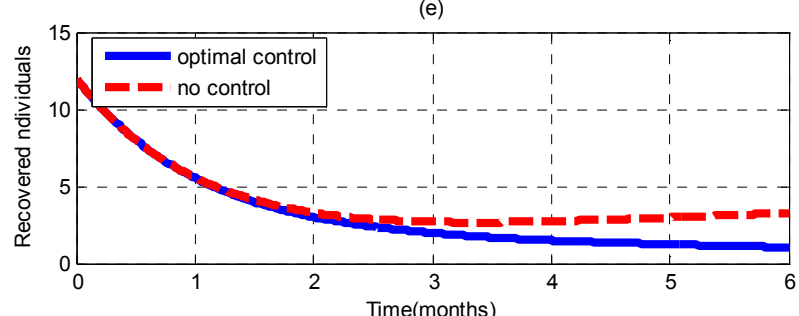

(f)

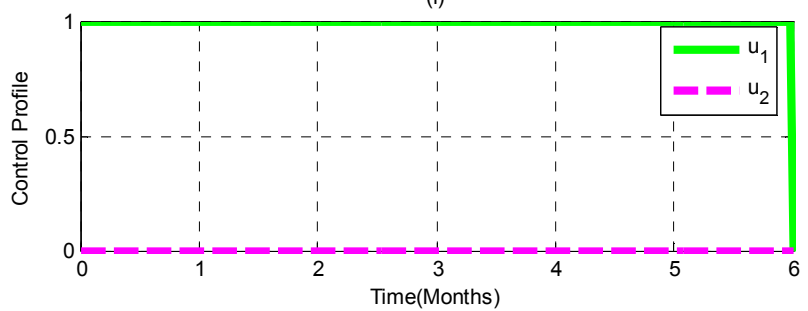

Figures 3. Simulation of the objective function for Ebola virus disease when only a control measure $\left(u_{1}\right)$ due to quarantine of exposed and susceptible individuals is applied.

Therefore by quarantining the exposed and susceptible population only, there will be reduction of disease persistence in the population, due to reduction of population in exposed and infected classes.

Figures 4 below, show the simulation of control problem (23), when only the control measure due to efficacy of drug

used for treating Ebola virus disease victim $\left(u_{2}\right)$ is optimized.

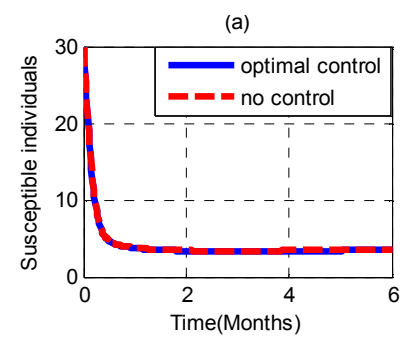

(c)
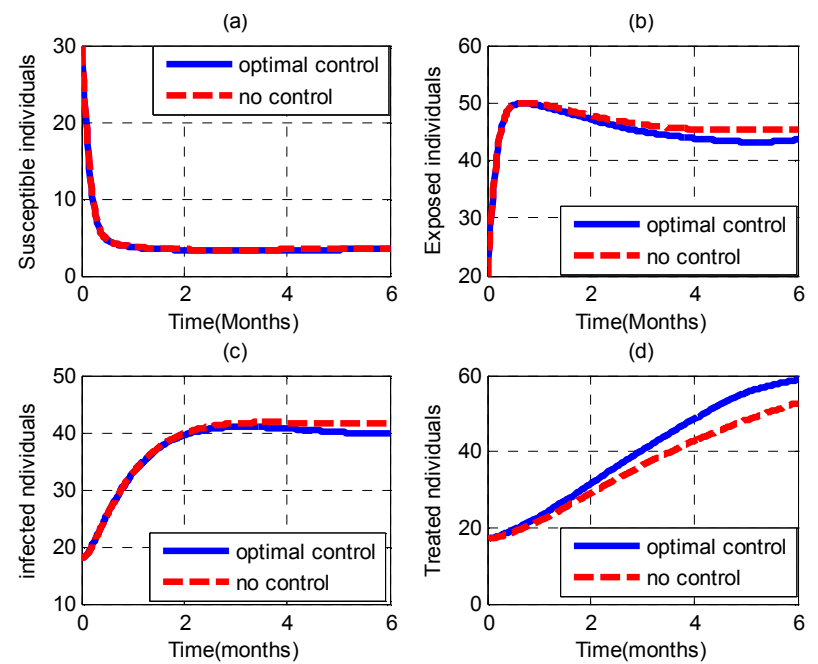

(d)

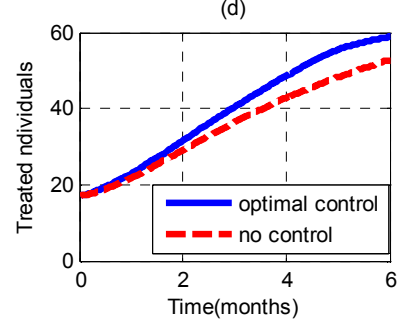

(e)

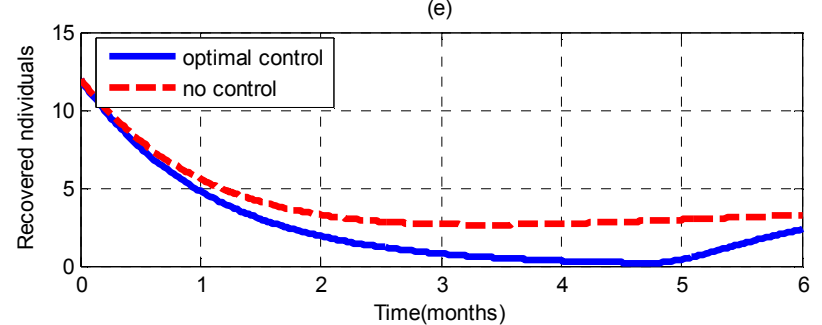

(f)

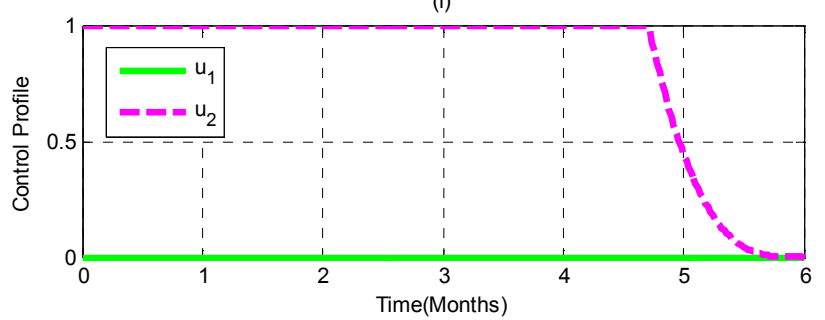

Figure 4 Simulation of control problem (23), when only the control measure due to efficacy of drug used for treating Ebola virus disease victim is optimized

Figure 4(a) shows that with one control, there will be no changes in susceptible population with time. Figures 4(b) and figure 4(c) show that there will be a slight reduction in exposed and infected populations with time. Figure 4(d) shows that there will be an increase in treated population with time. Figure 4(e) shows that there will be a decrease of recovered population with time due to the decrease in infected population with time.

Therefore optimizing the control measure due to efficacy of drug used for treating Ebola virus disease victim minimizes infections by reducing exposed and infected population while maximizing treatment.

Figure 5 below shows the simulation of control problem (23), when both controls $u_{1}$ (control measure due to quarantine of exposed and susceptible individuals) and $u_{2}$ (control measure due to efficacy of drug used for treating Ebola virus disease victim) are employed. 

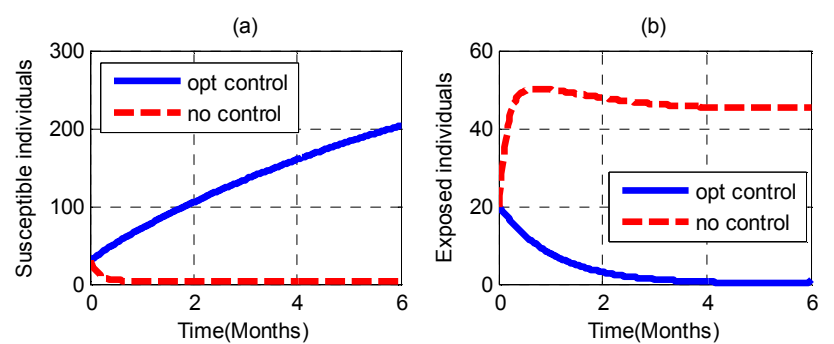

(c)
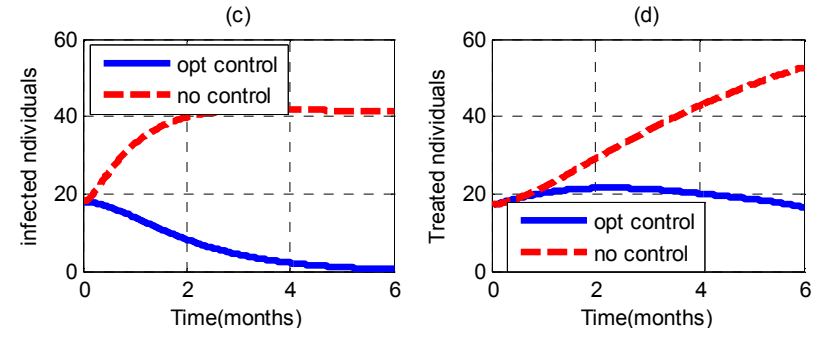

(e)

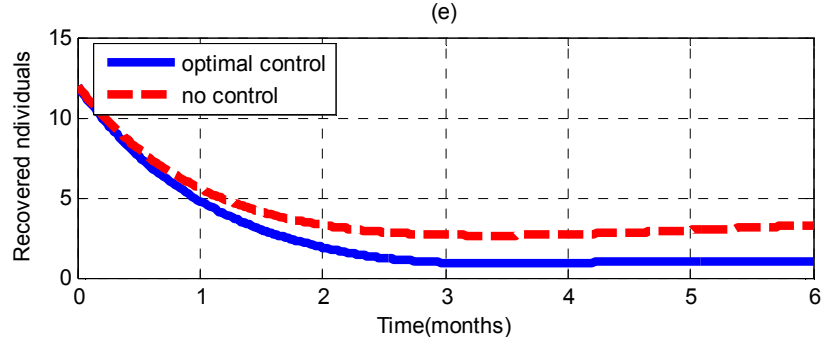

(f)

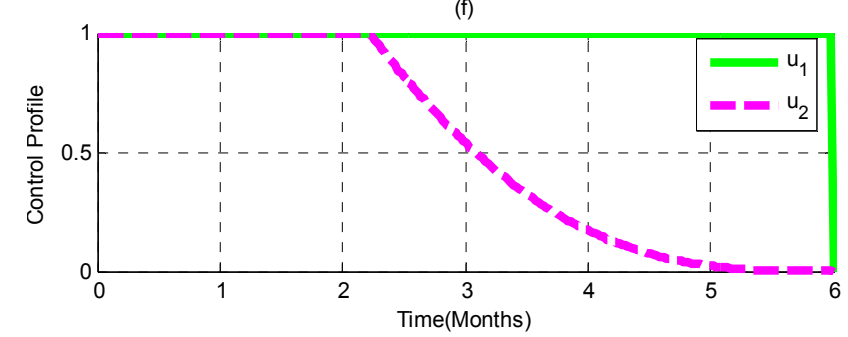

Figure 5. Simulation of control problem (23), when both controls are employed.

When both controls are employed, figure 5(a) shows that susceptible population increases with time. Figures 5(b) and figure 5(c) show that exposed and infected populations decrease with time respectively due to the decrease of number of people who acquire new infections. Figure 5(d) shows that treated population decreases with time due to reduction of the infected population. Figure 5(e) shows that recovered population are decreases with time due reduction of treated population with time. Figure 5(f) shows the control profile whereby $u_{1}$ (the control measure due to quarantine of exposed and susceptible individuals) remains at maximum up to the terminal time and $u_{2}$ (control measure due to efficacy drug used for treating Ebola virus disease victim) remains at maximum for at least two months before finally decreasing exponentially to the minimum.

From the simulations of the objective function, it can be seen that optimizing a control measure due to quarantine of exposed and susceptible individuals only $\left(u_{1}\right)$ has a greater effect in reduction of Ebola virus disease than using a control measure due to efficacy drug used for treating Ebola virus disease victim only $\left(u_{2}\right)$, since optimizing $u_{1}$ only reduces the exposed and infected population more than optimizing $u_{2}$ only. Also the simulations show that optimizing both the two controls $\left(u_{1}\right.$ and $\left.u_{2}\right)$ have a significant impact in reduction of disease transmission.

\section{Conclusions}

A mathematical model for the Ebola virus disease in the absence of control measures was developed and analysed. Using Routh Hurwitz criteria, it was established that the disease free equilibrium is locally asymptotically stable whenever the reproduction number is less than one $\left(R_{0}<1\right)$. The endemic equilibrium was determined and found to be locally asymptotically stable whenever the reproduction number is greater than one $\left(R_{0}>1\right)$. By applying two controls, i.e. treatment and Quarantine of individuals and efficacy of treatment drug used for treating Ebola virus disease victims, the optimal control problem was analysed so as to minimize the infections of Ebola Virus disease, while optimizing the controls. The control problem was finally simulated in order to examine the effect of one control or both controls in controlling Ebola virus disease. It was found that, optimizing a control measure due to quarantine of exposed and susceptible individuals only is more effective in reduction of Ebola virus disease than using a control measure due to efficacy drug used for treating Ebola virus disease victim only. However, simulations further showed that optimizing both of the two controls have a significant impact in reduction of disease transmission.

\section{References}

[1] Ustun C and Ozguller O 2004, Ebola: As an infectious Disease and as a potential Bioterrorism agent, Turk J med sci. 35(2005) $1-4$

[2] World Health Organisation (WHO), 2014, Ebola and Marburg Virus Disease Epidemics; Preparedness, alert, control and Evaluation. WHO Press; Geneva, Switzerland, www.paho.org/hq/Index.Php? option $=$ com_docman\&task $=$ doc_view\&gid $=2416 \&$ Itemid

[3] Birmingham K and Cooney S, 2002, Ebola: Small but real progress (News feature) Nature med, 8(4), 313

[4] Sriram, 2014. An Overview on Ebola Virus Disease (EVD) or Ebola hemorrhagic Fever (EHF), International Journal of Allied Medical Sciences and clinical Research (IJAMSCR), $269-278$

[5] Mupapa K, Massamba M, Kibadi K, Kuvula K, Bwaka A, Kipasa M, Colebunders R and Muyembe JJ, 2001. Treatment of Ebola hemorrhagic fever with blood transfusions from convalescent patients. The Journal of infectious diseases association of America 1999;179 (supp 1); S 18-23

[6] Ki M, 2014. What do we really fear? The epidemiological characteristic of Ebola Virus and our preparedness. Korean Society of Epidemiology. Volume 36, Article ID: e2014014

[7] Rodriguez HS, Monteiro MT and Torres DF, 2014. Optimal Control and Numerical Software: an Overview. Nova Science Publishers, New York, 93-110 
[8] Gaff H and Schaefer E, 2009. Optimal Control applied to Valuation and Treatment Strategies for Various Epidemiological models, pubmed. Jul; 6(3): 469-492

[9] Gumel AB and Lenhart S, 2010. Modelling Paradigms and analysis of Disease Transmission Models, American Mathematical Society, New York, United States of America

[10] Massawe LN (2015 Modelling Infectiology and Optimal Control of Dengue Epidemic, Applied and Computational Mathematics 2015; 4(3): 181-191

[11] Chowell G, Hengartner NW, Castillo-Chavez C, Fenimore PW, Hyman JM: The basic reproductive number of Ebola and the effects of public health measures: the cases of Congo and Uganda. J Theor Biol. 2004, 229: 119-126. 10.1016/j.jtbi.2004.03.006

[12] DiekMann O, Heesterbeek, JAP and Roberts MG, 2010, The construction of next generation matrices for compartmental epidemic models, J. R. Soc Interface, 7(47):873-885

[13] Diekmann O, Heesterbeek JAP and Metz, JAJ, on the definition and computation of the basic Reproduction ratio, In models for infectious diseases in heterogeneous population, Journal of Mathematics Biology, 28(1990):365-382
[14] Brauer F, Driessche VP and Wu J, 2008, mathematical epidemiology, Springer - verlag Berlin Heidelberg, Heidelberg, Germany

[15] Cai L and Li Z, 2010, analysis of a simple vector-host epidemic model with direct transmission, xinyang university, Henan, China

[16] Lenhart S and Workman JT, (2007). Optimal control, Applied to Biological Models, Chapman \& hall/CRC, London, United Kingdom

[17] Makinde OD and Okusun KO, 2013, Optimal control analysis of Malaria in the presence of Non-Linear incidence rate, Appl. Comp. Math, 12(1); 20-32

[18] Rivers CM, Lofgren ET, Marathe M, Eubank S and Lewis BL, 2014. Modelling the impact of interventions on an epidemic of Ebola in Sierra Leone and Liberia. Plos current outbreaks, fd 38 dd 8507856540bobe3fcd78f5ccf 